\title{
Editorial
}

\section{Blood Cholesterol Treatment: The National Cholesterol Education Program (NCEP) Adult Treatment Panel (ATP) Guideline}

(Birdem Med J 2015; 5(2): 68-72.)

The National Cholesterol Education Program (NCEP) Adult Treatment Panel (ATP) guideline is the national guideline for management of dyslipidemia in adults. Previously, ATP III guideline was released in 2001. ATP IV was originally scheduled for release in 2009, but there have been many factors involved with the delays.

The journey of ATP guidelines for the management of dyslipidemia till date is as follows:

ATP I : : LDL-C target was set to $<130 \mathrm{mg} / \mathrm{dl}$, focus was more on secondary prevention.

ATP II : targeted low-density lipoprotein cholesterol (LDL-C) to $<100 \mathrm{mg} / \mathrm{dl}$, focus was on secondary prevention, highdensity lipoprotein cholesterol (HDL-C) $<35 \mathrm{mg} / \mathrm{dl}$ was introduced as coronary heart disease (CHD) risk. TG (triglyceride) $200 \mathrm{mg} / \mathrm{dl}$ was taken as normal.

An update of ATP II was released in 2001 with 4 important modifications -

1. TG goal was reduced to $150 \mathrm{mg} / \mathrm{dl}$, HDL cut point was raised.

2. “CHD risk equivalent” was introduced (Diabetes mellitus and patients with >20\% CHD 10 year risk, based on Global risk was score, Framingham Heart study).

3. Fasting and random sugar was used to identify further cases at risk and stratify treatment.

4. Non HDL was identified as a secondary target for those with elevated TG>200 mg/dl and metabolic syndrome.

Life habit modification programme was introduced.

ATP III: A target of LDL-C $<70 \mathrm{mg} /$ dl was introduced in extremely high risk patients.
LDL-C drug treatment is indicated in addition to TLC (Therapeutic life style chane) if LDL-C levels $>100$ $\mathrm{mg} / \mathrm{dl}$ (previously only for LDL-C > $130 \mathrm{mg} / \mathrm{dl}$. initiation of an LDL-C lowering drug to achieve a level of $<100$ $\mathrm{mg} / \mathrm{dl}$ was a therapeutic option if patient baseline LDLC level or after TLC was 100-129 mg/dl. The intensity of LDL lowering drug treatment in high risk and moderately high risk patient must be sufficient to achieve at least a $30 \%$ to $40 \%$ reduction in LDL level beyond TLC.

ATP IV: This guideline for cholesterol management was introduced in 2013. It introduced atherosclerotic cardiovascular disease (ASCVD) risk reduction by focusing on 4 statin benefit groups.

Other organizations and their contributory efforts:

1. ADA (American Diabetic Association) / ACCF (American College of Cardiology Foundation):

Their consensus statement on Lipoprotein management in patients with cardio metabolic risks recommended more stringent lipid goals for patients with cardio metabolic risk (HTN, obesity, IR, abnormal lipid metabolism, family history, inflammation/hypercoagulability). For very high risk LDL $<70$, non HDLC $<100$, Apo B <80; High risk LDL $<100$, Apo $B<90$ (similar to optional goals from 2004 ATP III updates). Expanded statin use was recommend in DM with CHD regardless of baseline lipids . and for DM > 40 with I risk factors. AHA (American Heart Association) / ACC (American College of Cardiology).

In their guideline they recommend secondary prevention for patients with ASVD. They also stated that LDL goal $<70$ is reasonable for very high risk patients. They planned to review their recommendation in light of the ATP guidelines. 
2. AACE (American Association of Clinical Endocrinology).

Their guideline "Management of Dyslipidemia and Prevention of Atherosclerosis" was released in 2012. They recommended a lower LDL goal for very high and high risk groups and patients with pre-diabetes to have same treatment goals as diabetic patients.

Introduced FBS or random sugar (RS) to stratify risk, support use of apo B or LDL particle number to further assess residual risk in patient with TG $>150 \mathrm{mg} / \mathrm{dl}$ and $\mathrm{HDL}<40$.

Flashback on ATP III

ATP III classification of cholesterol concentration

\begin{tabular}{lll}
\hline Lipoprotein & Concentration(mg/dl) & Interpretation \\
\hline TC & $<200$ & Desirable \\
& $200-239$ & Borderline high \\
& $>240$ & High \\
LDL-C & $<100$ & Optimal \\
& $100-129$ & Near/Above \\
& & normal \\
& $130-159$ & Borderline high \\
& $160-189$ & High \\
& $>190$ & Very high \\
HDL-C & $<40$ & Low* \\
& $>60$ & High \\
TG & $<150$ & Normal \\
& $150-199$ & Borderline high \\
& $200-499$ & High \\
& $>500$ & Very high \\
\hline
\end{tabular}

*In ATP II \& ATP III, low HDL was $<35$ and $<40$ respectively.

\section{ATP III treatment targets:}

- To reduce CV risk and reduce CHD events.

- Primary target was LDL-C.

- Secodary target was HDL-C (once LDL is met and if fasting TG>200) .

- If fasting TG $>500$, it became primary target as it causes pancreatitis.

2013 ACC/AHA Blood Cholesterol Treatment ATP IV Guideline:

(2013 ACC/AHA Guideline on the Treatment of Blood Cholesterol to Reduce Atherosclerotic Cardiovascular Risk in Adults- A Report of the American College of
Cardiology/American Heart Association Task Force on Practice Guidelines)

Scope of guideline:

1. To reduce atherosclerotic cardiovascular (ASCVD) risk.

2. ASCVD-coronary heart disease (CHD), stroke and peripheral arterial disease, all of presumed atherosclerotic origin.

3. To provide strong evidence based foundation.

4. Only evidence from statin RCTs were used to develop guidelines

5. Comprehensive approach to lipid management for purposes with relation to ASCVD reduction only, not for complex lipid disorders.

\section{What is new in the guideline:}

a) Focus on ASCVD risk reduction: 4 statin benefit groups.

b) A new perspective on LDL-C and/or Non HDL-C treatment goals.

c) New method of estimate of 10 year ASCVD risk.

d) Identification of patients who is not benefitted.

e) Statin adverse effect documentation from RCT.

f) Treatment decision process for patients who are not included in 4 benefit groups.

\section{Benefits of statins:}

1. High intensity therapy - lowering LDL cholesterol by $>50 \%$.

2. Moderate intensity therapy - lowering LDL cholesterol by 30-50\%.

3. Reduces ASCVD events across the spectrum of baseline LDL-C levels > 70mg/dl

4. Relative reduction in ASCVD risk is consistent for primary and secondary prevention .

5. Absolute reduction in ASCVD events in proportional to baseline ASCVD risk.

6. Statin therapy only for individuals at increased ASCVD risk.

ASCVD - atherosclerotic cardiovascular disease:

Patients with history of myocardial infarction, stable or unstable angina, coronary revascularization, stroke or transient ischemic attack of atherosclerotic origin and peripheral arterial disease or revascularization. 
Statin benefit groups for ASCVD primary prevention: who are to be benefited by statin?

Recommendation:

High or moderate intensity statin therapy for all who will benefit from ASCVD risk reduction with life style modification before and during statin therapy. The benefit of ASCVD risk reduction outweighs the risk of adverse effect from statin therapy.

\section{ASCVD statin benefit group 1:}

This group includes adults with clinical ASCVD requiring secondary prevention.

- High intensity statin therapy is recommended for those $\geq 75 y$ rs. This reduces LDL-C cholesterol by $>50 \%$.

- Moderate intensity statin therapy is indicated for those age $>75$ years or those who are not candidate for high intensity statin. This reduces LDL-C by $30-50 \%$.

\section{ASCVD statin benefit group 2:}

- $\quad$ Primary ASCVD prevention is recommended for those with LDL-C >190 mg/dl and familial hypercholesterolemia (candidate for statin therapy) with high intensity statin therapy. Candidates not tolerating high intensity therapy, moderate intensity is recommended.

\section{ASCVD statin benefit group 3:}

- $\quad$ Adults aged 40-75 yrs with diabetes and LDL-C 70$189 \mathrm{mg} / \mathrm{dl}$ are candidates for moderate intensity statin therapy for ASCVD primary prevention.

- In those with estimated 10 yrs risk $>7.5 \%$, high intensity statin therapy is indicated.

\section{ASCVD statin benefit group 4:}

- $\quad$ These are the adults aged 40-75 years with LDL-C 70-189 mg/dl without diabetes mellitus and estimated 10-yr ASCVD risk > 7.5\%.

- $\quad$ ASCVD primary prevention is recommended for them with moderate to high intensity statin therapy.

High- Moderate- and Low-Intensity Statin Therapy (Used in the RCTs reviewed by the Expert Panel)*

\begin{tabular}{|c|c|c|}
\hline High-Intensity Stain Therapy & Moderate-intensity Stain Therapy & Low-intensity stain therapy \\
\hline Daily dose lower LDL-C on & Daily dose Lowers LDLC on & Daily dose lowers LDL-C on \\
\hline \multirow[t]{2}{*}{ average, by approximately > 50\% } & average, by approximately 30\% & average, by $<30 \%$ \\
\hline & to $50 \%$ & \\
\hline Atorvastain (40†) & Atorvastain 10 (20) mg & Simvastain $10 \mathrm{mg}$ \\
\hline \multirow[t]{7}{*}{ Rosuvastain 20 (40) mg } & Rosuvastain (5) $10 \mathrm{mg}$ & Pravastain 10-20 mg \\
\hline & Simvastatin 20-40 mg $\ddagger$ & Lovastain 20mg \\
\hline & Pravastain 40 (80) mg & Flvastain $20 \mathrm{mg}$ \\
\hline & Lovastain $40 \mathrm{mg}$ & Pitavastain $1 \mathrm{mg}$ \\
\hline & Fluvastain XL 80 mg & \\
\hline & Fluvastain $40 \mathrm{mg}$ bid & \\
\hline & Pitavastatin 2-4 mg & \\
\hline
\end{tabular}

Statins and doses that are approved by the U.S. FDA but were not tested in the RCTs reviewed are listed in italics.

*Individual responses to statin therapy varied in the RCTs and should be expected to vary in clinical practice. There might be a biologic basis for a less-than-average response.

†Evidence from 1 RCT only: down-titration if unable to tolerate atorvastatin $80 \mathrm{mg}$ in IDEAL (47).

¥Although simvastatin $80 \mathrm{mg}$ was evaluated in RCTs, initiation of simvastatin $80 \mathrm{mg}$ or titration to $80 \mathrm{mg}$ is not recommended by the FDA due to the increased risk of myopathy, including rhabdomyolysis.

bid indicates twice daily; FDA, Food and Drug Administration; IDEAL, Incremental Decrease through Aggressive Lipid Lowering study; LDL-C, low-density lipoprotein cholesterol; and RCTs, randomized controlled trials. 
Initiation and management of statin therapy for secondary ASCVD prevention among adults aged $>75$ years who have

a. Clinical ASCVD

b. Conditions or drug-drug interactions influencing statin therapy

c. A history of statin intolerance d.who are currently not on statin

1. Initial evaluation before initiating statin therapy :

a. Estimate Fasting blood lipid, ALT, CPK

b. Evaluate for secondary causes

c Evaluate and treat lab abnormalities influencing statin therapy.

TG> 500 mg/dl, LDL -C>190 mg/dl (secondary causes, screen for family history if primary) unexplained ALT > 3x Upper limit normal

2. Initiate moderate intensity statin therapy, counsel on lifestyle

3. Monitor statin therapy

Initiation and management of statin therapy for Primary ASCVD prevention among adults aged 40-75 years who have
a. no ASCVD
b. LDL -C 70-189 mg/dl
c. no diabetes
d. not on lipid lowering drug

1. Initial evaluation before initiating statin therapy:

a. Estimate Fasting blood lipid, ALT, CPK

b. Evaluate for secondary causes

c. Evaluate and treat lab abnormalities influencing statin therapy.

TG> 500 mg/dl, LDL -C > 190 mg/dl(secondary causes, screen for family history if primary) unexplained ALT $>$ 3x upper limit normal

2. Assign to statin benefit group 4, counsel on lifestyle

3. Estimate 10-yr ASCVD risk with pooled cohort equations-(7.5\%, 5 to $<7.5 \%,<5 \%)$ and a.discuss with patient about ASCVD risk reduction benefit, drug adverse effect, consider patient preference, consider additional factors to inform treatment decision to select patient.

4. Initiate statin therapy based on statin benefit group assignment and reinforce need for healthy lifestyle.

5. Monitor statin therapy.
Initiation and management of statin therapy for Primary ASCVD prevention among adults aged 40-75 years with diabetes or LDL LDL-C > $190 \mathrm{mg} / \mathrm{dl}$ who have no ASCVD who are not on lipid lowering drug:

1. Initial evaluation before initiating statin therapy :

a. Estimate Fasting blood lipid, ALT, CPK, HbA1C

b. Evaluate for secondary causes

c. Evaluate and treat lab abnormalities influencing statin therapy.

TG> 500 mg/dl, LDL -C > 190 mg/dl(secondary causes, screen for family history if primary) unexplained ALT > 3x upper limit normal

2. Assign to statin benefit group3: Diabetes and age 40-75 years OR LDL -C > $190 \mathrm{mg} / \mathrm{dl}$ and counsel on

\section{Lifestyle}

3. Discuss with patient about ASCVD risk reduction benefit, drug adverse effect, consider patient preference, consider additional factors to inform treatment decision to select patient.

4. Initiate statin therapy based on statin benefit group assignment and reinforce need for healthy lifestyle.

5. Monitor statin therapy

Primary ASCVD prevention in individuals with diabetes mellitus:

Moderate intensity statin therapy is recommended for all individuals aged $40-75$ years with diabetes.

High intensity statin therapy is recommended for all individuals aged $40-75$ years with diabetes and 10 years ASCVD risk > 7.5\%.

Individuals aged 40-75 years with diabetes have substantially increased lifetime risk for ASCVD events and death. Greater morbidity and worse survival after ASCVD onset are seen in these individuals.

It is recommended that statin therapy be individualized based on ASCVD reduction benefits, potential for adverse effects and drug-drug interactions and patient preferences.

Statin therapy for primary prevention for diabetes and LDL-C 70-189 mg/dl:

1. For adults aged 40-75 years with diabetes, use moderate-intensity statin therapy.

2. For adults aged 40-75 years with diabetes and estimated 10-years ASCVD risk >7.5\%, use high intensity statin therapy unless contraindicated. 
3. For adults aged $<40$ years or $>75$ years with diabetes evaluate the potential for ASCVD risk reduction benefits, adverse effects, drug-drug interactions and consider patient preference when initiating, continuing or intensifying statin therapy.

Estimate.d 10-yr ASCVD risk can be used to guide statin therapy for

1. ASCVD primary prevention in individuals with LDLC 70-189 mg/dl: guides initiation of statin

2. ASCVD primary prevention in individual with diabetes: guide statin intensity. It is not appropriate to estimate 10-yr ASCVD risk for individuals with clinical ASCVD or LDL-C >190 $\mathrm{mg} / \mathrm{dl}$ already in a statin benefit group.

10 year ASCVD risk assessment calculator primary prevention:

10-year ASCVD risk assessment calculator primary prevention is a comprehensive evaluation of 10 year risk for ASCVD events.

This includes coronary heart disease and stroke. The pooled cohort risk assessment equations are available at http://my.americanheart.org/icvriskcalculator.

The assessment calculator predicts stroke and CHD events for non Hispanic Caucasian and African American men and women aged 40-79 years with or without diabetes and LDL-C 70-189 mg/dl.

\section{LDL-C, non -HDL-C targets:}

No recommendation for or against specific LDL-C or Non--HDL-C targets for primary or secondary prevention has been made in this guideline.

There is a lack of evidence supporting titration of cholesterol lowering drug therapy to achieve optimal LDL-C or NoN-HDL-C level.
Primary ASCVD prevention for individuals not in a Statin benefit group:

This applies to adults with LDL-C $<190 \mathrm{mg} / \mathrm{dl}$ who are not identified in a statin group or adults for whom risk based treatment is uncertain after risk assessment.

Limitations of ATPIV:

- Clinical judgment required in patients, for whom RCT evidence is insufficient.

- Younger adults less than 40 years with Â7.5\% ASCVD risk for 10 years, high lifetime risk

- HIV patients, rheumatological patients

- $\quad$ RCTs, systemic reviews, meta-analysis of RCTs were taken into considation.

Future....

- Primary prevention in $>75$ years age

- Alternate treatment strategies

- Effectiveness of submaximal doses of statins vs nonstatins in intolarent patients

- Evaluation of the incidence of new onset diabetes associated with statin therapy.

- Outcomes of RCTs of new lipid modifying agents to determine the incremental ASCVD reduction when added to statin therapy.

Future updates required for....

1. The treatment of hypertriglyceridemia.

2. Use of non-HDL-C in decision making.

3. Whether on-treatment markers such as apo B, LDL particles are useful in guiding decisions.

4. Best approaches to use non-invasive imaging for refining risk estimates to guide treatment.

5. Optimal age for starting treatment for reducing lifetime risk of ASCVD.

6. What to do in patients with heart failure, hemodialysis.

7. Long term effects of statin associated new onset diabetes and management. 